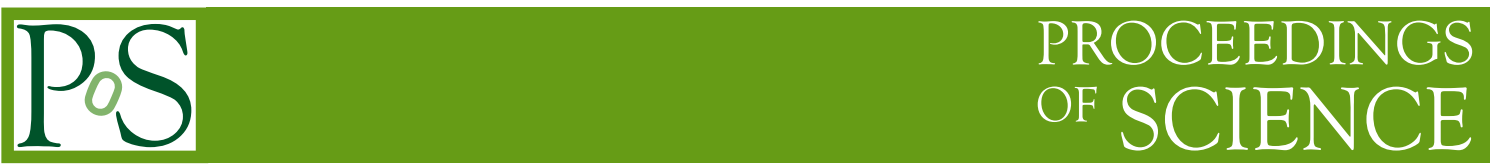

\title{
Omega-Omega interaction from 2+1 flavor QCD
}

\author{
Masanori Yamada for HAL QCD Collaboration* \\ Graduate School of Pure and Applied Sciences, University of Tsukuba \\ Tsukuba, Ibaraki 305-8571, Japan \\ E-mail: Sinvamadadhet.ph.tsukuba.ac.ip
}

\begin{abstract}
We report our results on the central potential between two Omega baryons in the 2+1 lattice QCD. Employing the HAL QCD method, we extract the Omega-Omega potential from the NambuBethe-Salpeter wave function, which encodes the scattering phase shift between Omega-Omega in its asymptotic behavior. In numerical calculations, we employed two gauge ensembles, one was generated by the CP-PACS/JLQCD Collaborations at L $=1.950(30)[\mathrm{fm}], m_{\pi}=871(1)[\mathrm{MeV}]$ and $m_{\Omega}=2104(8)[\mathrm{MeV}]$, and the other was generated by the PACS-CS Collaboration at L $=$ $2.902(42)[\mathrm{fm}], m_{\pi}=701(5)[\mathrm{MeV}]$ and $m_{\Omega}=1966(6)[\mathrm{MeV}]$. Using the potential obtained in lattice QCD, we calculate the phase shift of the Omega-Omega scattering and discuss a possibility for an existence of a shallow Omega-Omega bound state. Although an existence for the OmegaOmega bound state is inconclusive, we found that the Omega-Omega interaction is definitely strong attractive, showing that the system at this quark masses is in the unitary region.
\end{abstract}

32nd International Symposium on Lattice Field Theory LATTICE 2014

June 23, 2014 - June 28, 2014

NewYork, USA

* Speaker. 


\section{Introduction}

The nuclear potential is an important input for nuclear structure and astrophysical phenomena. It has been determined from experimental data plus some model calculations. In principle, however, one can derive the nuclear potential from the fundamental theory of strong interaction, QCD. Recently, a new but first-principle method, called the HAL QCD method, has been proposed to investigate nucleon-nucleon interactions in QCD on the lattice. The HAL QCD method can be applied not only to the nuclear potential but also to other interactions. For example, baryon-baryon interactions [1-3], meson-meson interactions [4, 5], hyperon-hyperon interactions [6-9], the LS force [10], the anti-symmetric LS force [11], and three body force [12], have been investigated. In addition, as an application of this approach, the structure of heavy nuclei and the equation of state for neutron stars have been investigated using the nuclear potential derived from the lattice QCD [13].

In this paper, we investigate the Omega-Omega interaction, as the first step to understand the the decuplet-decuplet interaction. Some model calculations predicted an existence of bound states in decuplet-decuplet systems. In particular, the Omega-Omega system is suitable to be studied, since the Omega baryon is stable in QCD, unlike other decuplet baryons.

For the Omega-Omega interaction in the $J=0$ channel, there have been several model calculations, which however seems contradicted with each other. Some predicted the strong attraction [14] but the other did the weak repulsive force [15]. In addition, the lattice QCD investigation on the Omega-Omega interaction by the finite volume method found the weak repulsion but with large errors [16]. Therefore, in this study, we try to determine a nature of the Omega-Omega interaction, attractive or repulsive, using the HAL QCD method.

\section{HAL QCD method}

We employ the HALQCD method to extract the Omega-Omega potential in lattice QCD. A key quantity in the HALQCD method is the NBS wave function, which encodes informations of scattering phase shifts in its asymptotic behavior [1-3].

The equal time NBS wave function concerned is defined by

$$
\psi_{n ; \alpha^{\prime} k^{\prime} \beta^{\prime} l^{\prime} ; \alpha \beta k l}\left(t, t_{0,} r\right) \equiv\left\langle 0\left|\Omega_{\alpha^{\prime}, k^{\prime}}(t, r) \Omega_{\beta^{\prime}, l^{\prime}}(t, 0)\right| \Omega_{\alpha, k}\left(k_{n}\right) \Omega_{\beta, l}\left(-k_{n}\right) ; i n\right\rangle,
$$

where $\mid \Omega\left(k_{n}\right) \Omega\left(-k_{n}\right)$; in $\rangle$ is a QCD asymptotic Omega-Omega in-state in the center of mass flame with the relative momenta $k_{n}$ and the total energy $E_{n}=2 \sqrt{m_{\Omega}^{2}+k_{n}^{2}}$. Local Omega operators, $\Omega(x)$ and $\bar{\Omega}$, are taken as

$$
\Omega_{\alpha, k}(x) \equiv \varepsilon^{a b c}\left[s_{a}^{T}(x) C \gamma_{k} s_{b}(x)\right] s_{c \alpha}(x), \quad \bar{\Omega}_{\alpha, k}(x) \equiv \Omega_{\alpha, k}^{\dagger} \gamma^{0}=\varepsilon^{a b c} \bar{s}_{a \alpha}(x)\left[\bar{s}_{b}^{T}(x) \gamma_{k_{1}} C \bar{s}_{c}(x)\right],
$$

where $a, b, c$ are color indices, $\varepsilon^{a b c}$ is the totally anti-symmetric tensor, $\gamma_{k}$ is the gamma matrix, and $\alpha$ is a spinor index. The charge conjugation matrix in the spinor space is taken as $C \equiv \gamma_{4} \gamma_{2}=$ $-C^{-1}=-C^{T}=-C^{\dagger}$ in Euclidean space-time. The Omega-Omega potential is defined through the following Schrödinger equation:

$$
\left(E_{n}-H_{0}\right) \psi_{n}(r)=\int d^{3} r^{\prime} U\left(r, r^{\prime}\right) \psi_{n}\left(r^{\prime}\right),
$$


where $H_{0} \equiv-\frac{1}{2 \mu_{\Omega}} \nabla^{2}$ is the non-interacting part of the Omega-Omega Hamiltonian, and $\mu_{\Omega} \equiv$ $m_{\Omega} / 2$ is reduced mass of the two-omega baryon system.

Note that the non-local but energy-independent potential $U\left(r, r^{\prime}\right)$ can be formally constructed as

$$
U\left(r, r^{\prime}\right)=\sum_{n, n^{\prime}}^{W_{n}, W_{n^{\prime}}<W_{\mathrm{th}}}\left(E_{n}-H_{0}\right) \psi_{n}(r) N_{n, n^{\prime}}^{-1} \psi_{n^{\prime}}^{*}\left(r^{\prime}\right),
$$

where $W_{\text {th }}$ is the threshold energy of the Omega-Omega system, and $N_{n, n^{\prime}}^{-1}$ is the inverse of the inner product matrix,

$$
N_{n, n^{\prime}} \equiv \int d^{3} r \psi_{n}^{*}(r) \psi_{n^{\prime}}(r), \quad W_{n}, W_{n^{\prime}}<W_{\text {th }} .
$$

Indeed it is easy to se that $U$ satisfies eq. ([2.3]) as

$$
\int d^{3} r^{\prime} U\left(r, r^{\prime}\right) \psi_{l}\left(r^{\prime}\right)=\sum_{n, n^{\prime}}\left(E_{n}-H_{0}\right) \psi_{n}(r) N_{n, n^{\prime}}^{-1} N_{n^{\prime}, l}=\left(E_{l}-H_{0}\right) \psi_{l}(r), \quad W_{l}<W_{\mathrm{th}} .
$$

In practice, we employ the time dependent method [17] to extract the potential as

$$
\left(\frac{1}{4 m_{\Omega}} \frac{\partial^{2}}{\partial t^{2}}+\frac{1}{m_{\Omega}} \nabla^{2}-\frac{\partial}{\partial t}\right) R\left(r, t, t_{0}\right)=\int d r^{\prime} U\left(r, r^{\prime}\right) R\left(r^{\prime}, t, t_{0}\right),
$$

where

$$
R\left(r, t, t_{0}\right) \equiv \frac{F\left(t, t_{0}, r\right)}{e^{-2 m\left(t-t_{0}\right)}}=\sum_{n} a_{n} \psi_{n}(r) e^{-W_{n}\left(t-t_{0}\right)},
$$

and $F\left(t, t_{0}, r\right)$ is the propagator of two Omega particles (i.e. the Omega 4-point correlation function). This method does not require the grand state saturation for the extraction of potentials [17].

In addition, the derivative expansion of the non-local potential is introduced at low energy. In this study, we keep only the leading-order term as

$$
U\left(\vec{r}, \vec{r}^{\prime}\right)=V(\vec{r}) \delta\left(\vec{r}-\vec{r}^{\prime}\right)+\mathscr{O}(\vec{\nabla})
$$

where $V(\vec{r})$ depends on quantum numbers of the Omega-Omega system. In our study, we fix both quantum numbers as explained in the next section.

\section{Projections}

Let us consider projection to the $J^{P}=0^{+}$state, where $J$ is the total angular momentum and $P$ is the parity, from which we extract the Omega-Omega potential. To fix $J$ and $P$, we consider the total spin $S$ and the orbital angular momentum $L$ for the Omega-Omega system.

We first consider the total spin. The decuplet baryon has spin and Lorentz vector indices. Since Omega baryon is heavy, we use non-relativistic operators for Omega as

$$
\begin{aligned}
\Omega_{\frac{3}{2}, \frac{3}{2}} & =-\left(\psi \Gamma_{+} \psi\right) \psi_{\frac{1}{2}} \\
\Omega_{\frac{3}{2}, \frac{1}{2}} & =\frac{1}{\sqrt{3}}\left[\sqrt{2}\left(\psi \Gamma_{Z} \psi\right) \psi_{\frac{1}{2}}+\left(\psi \Gamma_{+} \psi\right) \psi_{-\frac{1}{2}}\right] \\
\Omega_{\frac{3}{2},-\frac{1}{2}} & =\frac{1}{\sqrt{3}}\left[\sqrt{2}\left(\psi \Gamma_{Z} \psi\right) \psi_{-\frac{1}{2}}+\left(\psi \Gamma_{-} \psi\right) \psi_{\frac{1}{2}}\right] \\
\Omega_{\frac{3}{2},-\frac{3}{2}} & =\left(\psi \Gamma_{-} \psi\right) \psi_{-\frac{1}{2}}
\end{aligned}
$$


where $\Gamma_{ \pm} \equiv \frac{1}{2}\left(C \gamma^{2} \pm i C \gamma^{1}\right)$ and $\Gamma_{Z} \equiv \frac{-i}{\sqrt{2}} C \gamma^{3}$ are used to construct spin-1 di-quark operators and $\psi$ represents the strange quark operator. Using these operators, we construct the $S=0$ states as

$$
(\Omega \Omega)_{0,0}=\frac{1}{2}\left(\Omega_{\frac{3}{2}, \frac{3}{2}} \Omega_{\frac{3}{2},-\frac{3}{2}}-\Omega_{\frac{3}{2}, \frac{1}{2}} \Omega_{\frac{3}{2},-\frac{1}{2}}+\Omega_{\frac{3}{2},-\frac{1}{2}} \Omega_{\frac{3}{2} \cdot \frac{1}{2}}-\Omega_{\frac{3}{2},-\frac{3}{2}} \Omega_{\frac{3}{2} \cdot \frac{3}{2}}\right) .
$$

Secondly, we consider the projection of the orbital angular momentum. The wall-source automatically pick up the $L=0\left(A_{1}\right)$ state, while, for the sink operator, we employ the cubic group projection defined by

$$
P_{v}^{a}=\frac{d_{a}}{g} \sum_{i}^{g} D_{v v}^{a}\left(R_{i}\right)^{*} R_{i},
$$

where $a$ represents an irreducible representation of the cubic group, whose dimension is $d_{a}, R_{i}$ is an element of the cubic group and acts on $\vec{r}$ in the sink operator. $D^{a}\left(R_{i}\right)$ is the corresponding matrix in the irreducible representation acting on spin components, and $g$ is the order of the cubic group. We use the $A_{1}$ representation to pick up the $L=0$ state.

In our numerical calculation, we consider the Omega-Omega state with zero total spin ( $S=0$ ) and zero orbital angular momentum $(L=0)$, so that the total angular momentum and the parity, which are conserved, become zero $(J=0)$ and positive $(P=+)$.

\section{Numerical results}

\subsection{Simulation Set up}

In our numerical simulations, we have employed two ensembles of gauge configurations, both of which were generated by $2+1$ flavor QCD with the renormalization group improved gauge action and non-perturbatively $\mathscr{O}(a)$ improved Wilson quark action. The first ensemble, called the Set 1 , is consist of 700 gauge configurations generated by CP-PACS and JLQCD Collaborations [18] at $\beta=1.83(a \simeq 0.12 \mathrm{fm})$ on the $16^{3} \times 32$ lattice, whose physical extension becomes $L=1.92$ $\mathrm{fm}$, and at $\kappa_{u d}=0.13760, \kappa_{s}=0.13710$ corresponding to $m_{\pi}=875(1) \mathrm{MeV}$ and $m_{\Omega}=2104(8)$ $\mathrm{MeV}$. The second ensemble, called Set 2, contains 399 gauge configurations generated by PCASCS Collaborations [19] at $\beta=1.90(a \simeq 0.09 \mathrm{fm})$ on the $32^{3} \times 64$ lattice, whose physical extent becomes $L=2.9 \mathrm{fm}$, and at $\kappa_{u d}=0.13700, \kappa_{s}=0.13640$ corresponding to $m_{\pi}=701(5) \mathrm{MeV}$ and $m_{\Omega}=1966(6) \mathrm{MeV}$.

In both cases, to increase statics, we have employed 32 sources on different time slices per configuration as well as the rotational symmetry and the charge conjugation symmetry. The preliminary results using 9/32 of full statistics of Set 1 was reported in the last year [20]. All errors are estimated by the Jack-Knife method with the bin-size of 1 configurations.

\subsection{Omega-Omega Potential}

Fig. $\mathbb{W}$ show the central potentials for Omega-Omega in $J=0$ and $P=+$ channel. The left figure represents the potential obtained at $t-t_{0}=7,8,9$ on the Set 1 , while the right one show the potential at $t-t_{0}=11,12,13$ on the Set 2 . In both cases, the central potential has the repulsive core at short distance and strong attractive pocket at medium distance. We observe that $t$ dependence is negligible for the potential on the Set 2 but the potential at $t-t_{0}=9$ (red) on the Set 1 differs a litter from others, in particular at long distance. This $t$ dependence of the potential on the Set 1 might be caused by the finite size effect due to the smaller volume of the Set $1(L / 2=0.96 \mathrm{fm})$. 

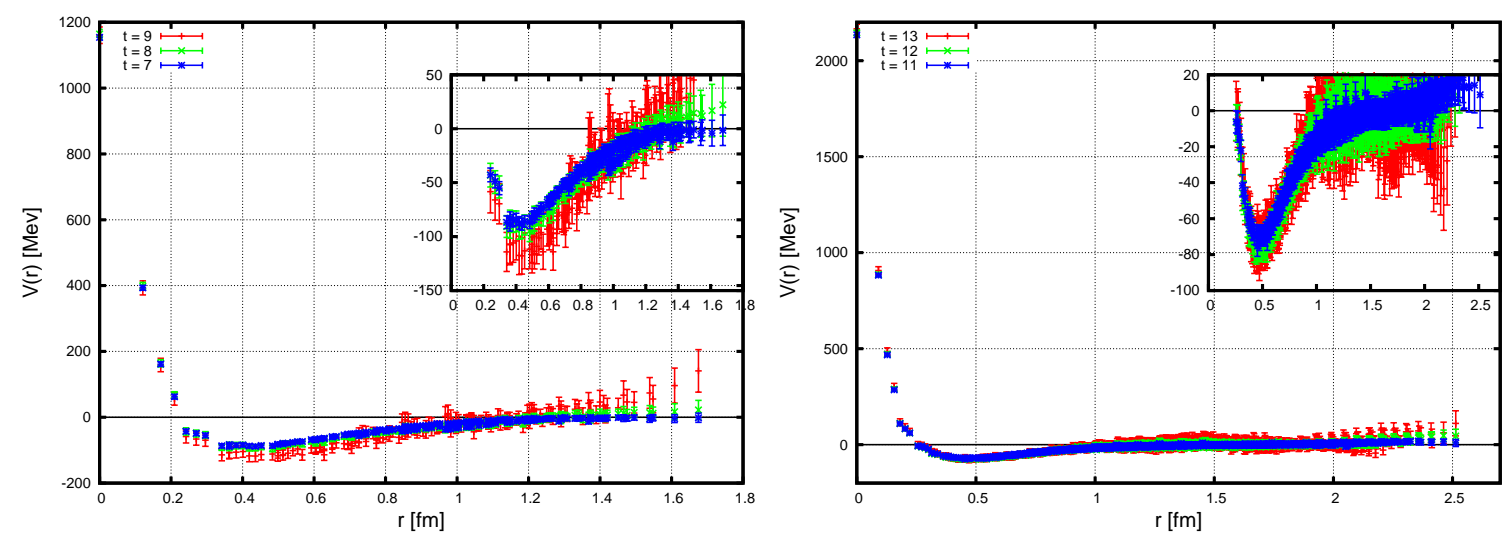

Figure 1: (Left) Central potential $V_{c}(r)$ for the Omega-Omega ${ }^{1} S_{0}$ state on the Set 1 at $t-t_{0}=7$ (blue), 8 (green), 9(red). (Right) The same but on the Set 2 at $t-t_{0}=11$ (blue), 12(green), 13(red).

\subsection{Phase shift and Scattering length}

We fit the potentials in Fig. Wusing the Gauss $+(\text { Yukawa })^{2}$ function given by

$$
V(r)=a_{1} e^{-a_{2} r^{2}}+a_{3}\left(1-e^{-a_{4} r^{2}}\right)^{2}\left(\frac{e^{-a_{5} r}}{r}\right)^{2}, \quad \lim _{r \rightarrow 0} V(r)=a_{1},
$$

or the 2 Gauss $+(\text { Yukawa })^{2}$ function given by

$$
V(r)=a_{1} e^{-a_{2} r^{2}}+a_{3} e^{-a_{4} r^{2}}+a_{5}\left(1-e^{-a_{6} r^{2}}\right)^{2}\left(\frac{e^{-a_{7} r}}{r}\right)^{2}, \quad \lim _{r \rightarrow 0} V(r)=a_{1}+a_{3} .
$$

For the potential on the Set 1 , since $\chi^{2} /$ dof is better, we have employed the Gauss $+(\text { Yukawa })^{2}$ function, and obtained $a_{1}=1.14(1) 10^{3} \mathrm{MeV}, a_{2}=62.9(1.8) \mathrm{fm}^{-2}, a_{3}=-523(434) \mathrm{MeV}, a_{4}=$ $2.21(83) \mathrm{fm}^{-2}, a_{5}=1.49(38) \mathrm{MeV}$ at $t-t_{0}=8$, while in the case of the Set 2, we have adopted the 2Gauss $+(\text { Yukawa })^{2}$ function, which gives $a_{1}=1.65(10) 10^{3} \mathrm{MeV}, a_{2}=125(400) \mathrm{fm}^{-2}, a_{3}=$ 480(107) MeV, $a_{4}=5.46(1.15) \mathrm{fm}^{-2}, a_{5}=-2.53(11.46) 10^{5} \mathrm{MeV}, a_{6}=3.76(7.09) 10^{-1} \mathrm{MeV}$, $a_{7}=3.62(40) 10^{-1} \mathrm{MeV}$ at $t-t_{0}=12$. We use these parameters to solve the Schrödinger equation in the infinite volume.

The phase shift $\delta(k)$, the scattering length $a$ and the effective range $r_{e}$ are extracted from the wave function as

$$
\tan \delta(k)=\lim _{x_{1}, x_{2} \rightarrow \infty} \frac{\psi_{k}\left(x_{2}\right) \sin \left(k x_{1}\right)-\psi_{k}\left(x_{1}\right) \sin \left(k x_{2}\right)}{\psi_{k}\left(x_{1}\right) \cos \left(k x_{2}\right)-\psi_{k}\left(x_{2}\right) \cos \left(k x_{1}\right)}, k \cot \delta(k)=\frac{1}{a}+\frac{1}{2} r_{e} k^{2}+\mathscr{O}\left(k^{4}\right),
$$

where $\psi_{k}$ is the wave function, $k$ is the corresponding momentum, and $a$ is the scattering length. The behavior of the phase shift in Fig. \ 1 suggests that the Omega-Omega system has a bound state in the ${ }^{1} S_{0}$ channel on the Set 1 . On the other hand, the phase shift on the Set 2 shows that the Omega-Omega system has strong attraction at low energy, which however is not strong enough to form a bound state.

For the Set 1, we have $a^{-1}\left(\mathrm{fm}^{-1}\right)=-2.1(1.1) \times 10^{-1},-1.6(0.9) \times 10^{-1},-9.3(3.5) \times 10^{-2}$ and $r_{e}(\mathrm{fm})=6.3(0.7) \times 10^{-4}, 6.1(1.2) \times 10^{-4}, 5.2(3.0) \times 10^{-4}$, at $t-t_{0}=7,8,9$, respectively. For the Set 2, We can't estimate the reasonable scattering length and effective range due to large error bar. 

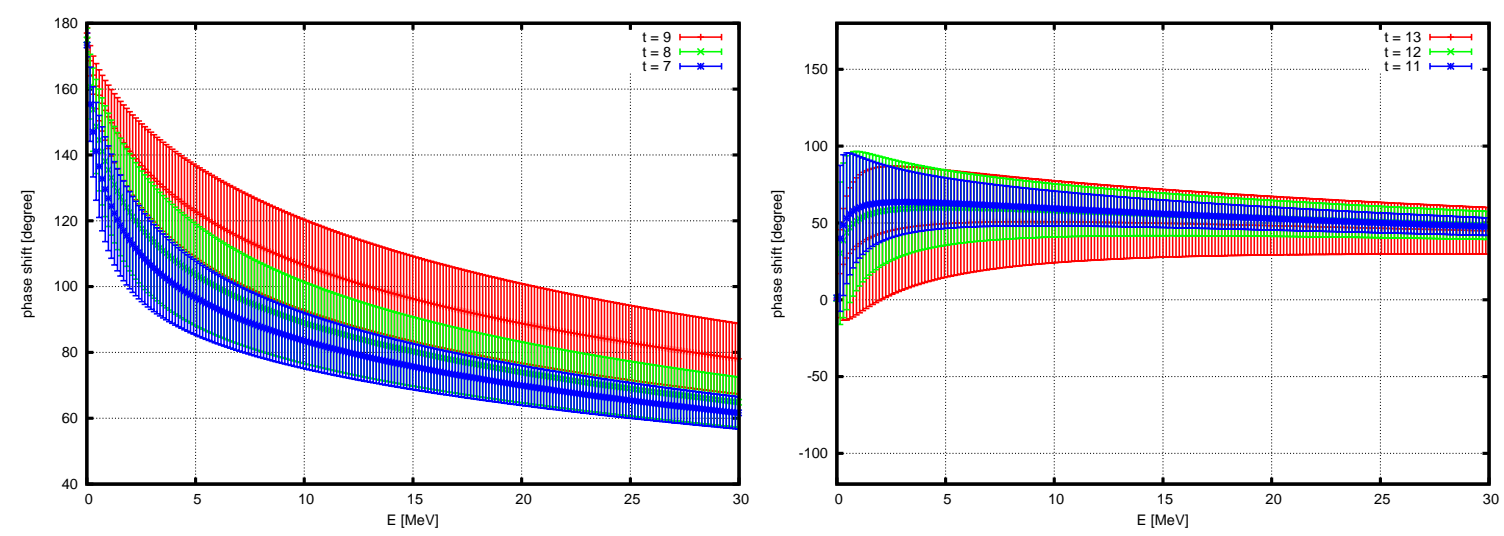

Figure 2: (Left) Phase shift $\delta(k)$ for the Omega-Omega ${ }^{1} S_{0}$ state on the Set 1 at $t-t_{0}=7$ (blue), 8 (green), 9(red). (Right) Same but on the Set 2 at $t-t_{0}=11$ (blue), 12(green), 13(red).

\subsection{Binding energy}

We define S-matrix by using wave function.

$$
\phi(r)=\frac{i}{2}\left(F_{l}(+k) \hat{h}_{l}^{(-)}(k r)-F_{l}(-k) \hat{h}_{l}^{(+)}(k r)\right), \quad S_{l}(k) \equiv \frac{F_{l}(-k)}{F_{l}(+k)}
$$

where $\hat{h}_{l}^{( \pm)}$is the Hankel function. We differentiate $\phi_{l}(r)$ in $r$ to obtain the $F_{l}( \pm k)$ and get S-matrix by solving the following equation at large $r$.

$$
\left(\begin{array}{c}
\phi_{l}(r) \\
\frac{\partial}{\partial r} \phi_{l}(r)
\end{array}\right)=\left(\begin{array}{cc}
\hat{h}_{l}^{(-)}(k r) & -\hat{h}_{l}^{(+)}(k r) \\
\frac{\partial}{\partial r} \hat{h}_{l}^{(-)}(k r) & -\frac{\partial}{\partial r}{\hat{h_{l}}}^{(+)}(k r)
\end{array}\right)\left(\begin{array}{c}
F_{l}(+k) \\
F_{l}(-k)
\end{array}\right),
$$

where both $\phi_{l}(r)$ and its derivative are calculated numerically. We determine an existence of the bound state from the divergence of the S-matrix at some $k$, which gives the binding energy. As a results, we find $E=0.96(1.06) \mathrm{MeV}, E=1.77(2.23) \mathrm{MeV}, E=6.69(6.81) \mathrm{MeV}$ at $t-t_{0}=7,8$, 9 , respectively, for the Set 1 . On the other hand, we did not find any bound state for the Set 2.

Although we find a bound state on the Set 1 at $t-t_{0}=7,8,9$, the binding energy is small and is almost consistent with zero within large errors.

\section{Summary}

In this paper, we have investigate the Omega-Omega interaction extracting the corresponding potential through the HAL QCD method. We found that the Omega-Omega potential obtained from the $J=0$ state is strongly attractive at low energy. Although whether the Omega-Omega bound state can be produced by this strong attraction or not is inconclusive in our study, we can definitely say that the Omega-Omega system at present quark mass is in the "unitary region", where the attraction is strong so that the sallow bound state just appears or is about to form.

In future studies, it is interesting to investigate whether the strong attraction found for the Omega-Omega system in this report remains at lighter quark masses. 


\section{Acknowledgements}

We thank CP-PACS/JLQCD Collaborations, PACS-CS Collaboration and ILDG/JLDG for providing us the $2+1$ flavor gauge configurations [21]. We also thank authors and maintainer of CPS++ [22], whose modified version was used in this study. Numerical computations of this work have been carried out by the KEK supercomputer system (BG/Q) and by machines at University of Tsukuba. This research is supported in part by JICFUS [23].

\section{References}

[1] N. Ishii, S. Aoki and T. Hatsuda, Phys. Rev. Lett. 99 (2007) 022001.

[2] S. Aoki, T. Hatsuda and N. Ishii, Prog. Theor. Phys. 23 (2010) 89 [arXiv:0909.5585 [hep-lat]].

[3] Reviewed in, S. Aoki et al. [HAL QCD Collaboration], PTEP 2012 (2012) 01A105 [arXiv:1206.5088 [hep-lat]].

[4] Bruno Charron, for HAL QCD Collaboration, [arXiv:1212.6333 [hep-lat]].

[5] Y. Ikeda, C. Buruno, S. Aoki, D. Takumi, T. Hatsuda, T. Inoue, N. Ishii, K. Murano, H. Nemura, K. Sasaki, Phys. Lett B. 729 (2014) [arXiv:1311.6214 [hep-lat]].

[6] H. Nemura, for HAL QCD Collaboration, PoS(Lattice 2011) 167.

[7] K. Sasaki et al. [HAL QCD Collaboration], PoS(LATTICE 2011), 173 (2011).

[8] T. Inoue et al. [HAL QCD Collaboration], Phys. Rev. Lett. 106, 162002 (2011).

[9] K. Sasaki, Few Body Syst. 54, 1109 (2013).

[10] K. Murano et al. [HAL QCD Collaboration], Phys. Lett. B 735, 19 (2014).

[11] N. Ishii, K. Murano, H. Nemura, K. Sasaki for HAL QCD Collaboration, PoS (LATTICE 2013$) 234$.

[12] T. Doi et al. [HAL QCD Collaboration], Prog. Theor. Phys. 127, 723(2012).

[13] T. Inoue et al. [HAL QCD Collaboration], Phys. Rev. Lett. 111, 112503 (2013), [arXiv:1307.0299 [hep-lat]].

[14] F. Wang ,J.-1. Ping,G.-h. Wu, L.-j.Teng, H.T.Goldman. Phys Rev C. 51 (1995) 3411.

[15] Z.Y. Zhang,Y.W.Yu,C.R.Ching,T.H.Ho, and Z.-D.Lu, Phys.Rev.C 61 (2000) 065204.

[16] M. Buchoff et al. Phys. Rev. D85 (2012) 09451, [arXiv:1201.3596[hep-lat]].

[17] N. Ishii et al. [HAL QCD Collaboration], Phys. Lett. B 712 (2012) 437 [arXiv:1203.3642 [hep-lat]].

[18] CP-PACS/JLQCD Collaborations: T. Ishikawa et al., Phys. Rev. D 78 (2008) 011502(R).

[19] PACS-CS Collaboration: S. Aoki, et al., Phys. Rev. D79 (2009) 034503.

[20] M. Yamada for HAL QCD Collaboration, PoS (Lattice 2013) 232.

[21] ILDG/JLDG http://www.lqcd.org/ildg/http://www.jldg.org.

[22] ColumbiaPhysicsSystem(CPS),http://qcdoc.phys.columbia.edu/cps.html

[23] Joint Institute for Computational Fundamental Science http://www.jicfus.jp/jp/ 